\title{
Indirect Approach to Continuous Time System Identification of Food Extruder
}

\author{
Liuping Wang *, Peter Gawthrop ${ }^{* *}$, Charlie Chessari ${ }^{* * *}$ \\ Tony Podsiadly ${ }^{* * * *}$ Angus Giles ${ }^{* * * *}$ \\ * Discipline of Electrical Energy and Control Systems \\ School of Electrical and Computer Engineering \\ RMIT University \\ Victoria 3000, Australia \\ ** Department of Mechanical Engineering \\ University of Glasgow, Glasgow, Britain \\ ${ }^{* * *}$ Food Science Australia, North Ryde, Sydney, Australia \\ **** Centre for Integrated Dynamics and Control \\ University of Newcastle \\ Australia
}

Corresponding author: liuping.wang @rmit.edu.au; Tel: 61-3-99252100

\begin{abstract}
A three-stage approach to system identification in the continuous-time is presented which is appropriate for day-to-day application by plant engineers in the process industry. The three stages are: data acquisition using relay feedback; non-parametric identification of the system step response; and parametric model fitting of the identified step-response. The method is evaluated on a pilotscale food-cooking extruder.
\end{abstract}

Keywords: Continuous time systems, relay feedback control, Least squares, frequency domain, state variable filters, time domain

\section{Introduction}

The food-cooking extruder is an important processing unit within the food manufacturing industry. However, product quality control is often open-loop and thus significant improvements to food quality and throughput can be expected by the use of closed-loop quality control. It has been shown previously that model-based predictive control can be successfully applied to this problem (for example Wang et al.(2001)). It is known that to design such a control system, a suitable model of the food-cooking extruder is required. Unfortunately, because the extrusion cooking process has strong interactions between mass, energy and momentum transfer, coupled with complex physicochemical transformations, it is hard to derive a mathematical model from first principles. Moreover, 
such models can be overly complex for the purposes of model-based predictive control. For these reasons, the use of system identification to deduce a suitable model from input-output data is a more practical approach to building mathematical models of the food-cooking extruder suitable for model-based predictive control.

It is our experience that whilst process engineers in the food industry have a good intuitive grasp of the processes based on their engineering knowledge and experience, they have little experience of system identification techniques. For this reason, system identification will only be accepted in the process industries if each stage has a transparent result that relates directly to the knowledge and experience of industrial process engineers. This paper presents a three stage approach to system identification and demonstrates that each stage is both simple to apply and transparent in its results. The three stages are:

Data Acquisition using Relay Feedback: An automated technique for experimental data acquisition based on the relay feedback approach of Åström and Hagglund (1984), but with modified periodic oscillations (Wang et al., 1999).

Step Response using Frequency-sampling Filters: Identification of the system step response from the experimental data using the Frequency-sampling Filter (FSF) approach of Wang and Cluett (1997).

Continuous-time Transfer-function Identification: Identification of a (continuous-time) transfer function from the identified step response.

Each stage is automated, yet the output of each stage is readily understandable and can be examined by the process engineer before proceeding to the next stage. Thus the first stage yields data corresponding to square waves at the correct frequency to yield useful information and the process engineer can adjust input and output amplitudes according to his knowledge of process behaviour. The second stage gives a step response which is much 'cleaner' (in terms of noise and disturbance) than that obtained by a simple step response experiment and therefore can be matched to the experience and intuition of the engineer. The third stage yields a transfer function approximation to the step; the order of the transfer function can be chosen by the process engineer to trade off accuracy against complexity whilst yielding numerical values for steady-state gains, time constants, natural frequencies and damping.

One of the essential ideas behind the proposed approach is associated with the idea of 'data compression' in which the process experimental data using binary input signals are compressed into step responses. During this compression process well-established system identification tools and methods in discrete systems can be applied to obtain high quality step response models. High quality step response models with little noise will lead to the estimation of continuous time transfer function models with high accuracy(as demonstrated in this paper). In addition, the number of data points contained in a step response model is far less than the number of data points in a set of process experimental data using a binary input signal, which is inevitably advantageous in numerical computation of a continuous time transfer function model. It is worthwhile to point out that use of state-variable filters in the estimation of a continuous time transfer function model is essential for overcoming the well-known lack of excitation problem (Ljung, 1987) when a step input signal is used.

There was an attempt to identify a continuous time transfer function model of food extruder directly from input and output data using state-variable filter approach (Wang et al., 2001). However, because of the high noise level existed in the measurement of food extruder, it was numerically sensitive for the estimation of the pole locations in the continuous time model, as well as for the choice of state variable filters, even though a large number of experimental data were used. In contrast, in 
the proposed approach as the continuous time system estimation is set on the second stage of the estimation problem, it is anticipated that higher quality continuous time models will be obtained when there is little noise in the step response data.

Although none of these stages proposed in the paper are novel, the contribution of the paper is to combine them in a novel way and to verify the approach in an industrial application context. Section 2 considers the first stage; Section 3 considers the second stage and Section 4 considers the third stage. Section 5 contains the experimental evaluation of the procedure and Section 6 draws some conclusions.

\section{Data Acquisition using Relay Feedback}

A simple relay is a nonlinear element that switches between the levels $-a$ and $+a$ based on the error signal $e$ and generates a square wave input signal $u$ to the process. In the extruder case, the process outputs are corrupted with noise, hysteresis is added to the relay to reduce the effect of the noise (see Figure 1). Adding hysteresis to the relay produces a dead-zone to prevent the relay signal from switching due to the noise. It is well known that if the width of the hysteresis $\epsilon$ equals zero, then the oscillation frequency corresponds to the cross over frequency of the process under the feedback control. An integrator in series to the relay element generates a stable oscillation with the dominant frequency corresponding to $-90^{\circ}$ on the Nyquist plot (Åström and Hagglund, 1984).

A standard relay experiment produces in most cases a limit cycle dominated by a single frequency. However, this information is not sufficient for the estimation of a continuous time transfer function model. The strategy we adopt in the identification experiment design was introduced in Wang et al. (1999) and applied by Wang and Gawthrop (2001) to simulation studies of continuous time system identification, in which we make use of multiple relay experiments to generate frequency response information at several frequencies. The proposed apparatus combines in parallel a relay element with an integrator in series with a relay element. Figure 1 provides a block diagram of this apparatus. The experiment is performed by alternatively switching the error signal between the relay path and the integrator-relay path. The design of the experiment then reduces to the selection of this switching sequence. The proposed relay experiment on its own provides some interesting ideas about how to design input signals for continuous time identification. One of the main benefits of the apparatus is that the design of an identification experiment suitable for obtaining a mathematical model has now been automated. In addition, choice of sampling rate can be set to near continuous measurement.

\section{Step Response Estimation using Frequency-sampling Fil- ters}

The step response of a system is a standard non-parametric representation, which reflects system time delay, gain and response time in an intuitive way. It is called non-parametric (in contrast to parametric) in the sense that the process dynamics is captured by a set of response coefficients, instead of description of process poles and zeros. Step response representation is invariant between system descriptions in continuous time and discrete time at the sampling instant. It can be derived through direct plant step response test at the situations where the plant is relatively disturbance-free and its response time is relatively short (Rake, 1980). However, in many engineering applications, plant step response is estimated from a set of plant input and output data generated from a more 
extensive plant experiment such as the proposed relay experiment above. In the estimation of step response, it is assumed that a discrete-time-domain finite impulse-response (FIR) model(Ljung, 1987) of the form below is used to describe a stable system through the input and output relation:

$$
y(k)=\sum_{m=0}^{N-1} h_{m} u(k-m)+v(k)
$$

where $y(k)$ and $u(k), k=0,1,2, \ldots$, are the set of plant input and output response data; $h_{m}$, $m=0,1,2 \ldots, N-1$ are the impulse response coefficients. Upon obtaining the estimated $h_{m}$, step response coefficients can be constructed as shown later on. The core technique here is to use an FIR model to describe the process. Supposing that the plant settling time is $T$ and the sample interval is $\Delta t$, the basic approach is to directly identify the $N=\frac{T}{\Delta t}$ coefficients $h_{m}$ corresponding to times $t=0, \Delta t, \ldots, T$. This approach has a number of disadvantages: in particular as $\Delta t$ gets small (fast sampling), the number of parameters gets large; and the estimated step is not smooth as each estimated parameter is a random variable.

A growing methodology in the field of system identification uses basis functions (see Wahlberg (1991), Ninness and Gustafsson (1997) and Wang and Cluett(2000)). The FIR approach mentioned above is a special case of the basis function approach where the (time-domain) basis functions are $\delta_{k}(i)$ where:

$$
\delta_{k}(i)= \begin{cases}1 & i=k \\ 0 & i \neq k\end{cases}
$$

The frequency domain equivalent is to represent the transfer function as a weighted sum of filters of the form:

$$
H_{f i r}^{l}=\frac{1}{z^{l}}
$$

with multiple poles at $z=0$.

The disadvantages of the FIR approach (particularly in the case when $N$ is large) can be removed by using a set of $n \ll N$ basis functions which are more sophisticated than those of (2) or (3). In particular we use the set of basis functions corresponding to the step response of a set of discrete-time Frequency-sampling filters (FSF) developed by Wang and Cluett(1997). As the name suggests, these filters have poles on the z-plane unit circle uniformly spaced by a frequency-domain sampling interval $\Omega=\frac{2 \pi}{N}$. In particular, $n$ is chosen to be an odd number and the filter poles are at: $1, e^{ \pm j \Omega}, e^{ \pm 2 j \Omega}, \ldots, e^{ \pm \frac{n-1}{2} j \Omega}$, which guarantees that the frequency sampling filters appear in complex conjugate pairs. Figure 2 shows the location of the filter poles when $n=9$ and $N=50$. More specifically the $l$ th discrete-time filter $H^{l}(z)\left(\right.$ with pole at $\left.e^{j l \Omega}\right)$ is given by:

$$
H^{l}(z)=\frac{1}{N} \frac{1-z^{-N}}{1-e^{j l \Omega} z^{-1}}
$$

and the set of $\mathrm{n}$ filters (with poles given by Figure 2) is given by (4) with $-\frac{n-1}{2} \leq l \leq \frac{n-1}{2}$. A system $G(z)$ with this set of poles can then be represented in partial fraction (pole/residue) form as the weighted sum of the set of filters where the weights are the complex residues of $G(z)$ at the lth pole:

$$
G(z)=\sum_{l=-\frac{n-1}{2}}^{\frac{n-1}{2}} G\left(e^{j l \Omega}\right) H^{l}(z)
$$


The $l t h$ frequency sampling filter can also be written in a complex geometric series expansion as

$$
H^{l}(z)=\frac{1}{N}\left(1+e^{j l \Omega} z^{-1}+e^{j 2 l \Omega} z^{-2}+\ldots+e^{j(N-1) l \Omega} z^{-(N-1)}\right)
$$

At $z=e^{j l \Omega}, H^{l}(z)=1$.

Note that (for $|l|>0$ ) the weights $G\left(e^{j l \Omega}\right)$ occur in complex conjugate pairs corresponding to the complex-conjugate filter poles of Figure 2. These first order, complex filters appearing in pairs can always be rewritten as an equivalent second-order, real, filters. Thus it is always possible to deal in real numbers only. However, as is usual in filter theory, it is simpler to develop the theory in first-order, complex, form. If $n=N$, the FIR model is a linear transformation of the FSF model (5). However, if $n<N$, the FSF filter representation is a low-pass filtered representation of the process as the filters corresponding to the points marked as $\diamond$ in Figure 2 have been removed. In the context of system identification (and assuming that $\frac{n-1}{2} \Omega$ is significantly larger than the system bandwidth), this has the useful effect of removing the corrupting effect of high frequency noise and gives a smooth estimated step response.

To be more precise, with description of the system using frequency sampling filters, it is assumed that the system response can be described by

$$
y(k)=\sum_{l=-\frac{n-1}{2}}^{\frac{n-1}{2}} G\left(e^{j l \Omega}\right) H^{l}(z) u(k)+v(k)
$$

for a suitable choice of $G\left(e^{j l \Omega}\right)^{\prime} s$, where $u(k)$ is the process input, $y(k)$ is the process output and $v(k)$ is the disturbance signal. Figure 3 shows the system input-and-output relationship using frequency sampling filter model. The output $y(k)$ can be expressed in a linear regression form by defining the parameter vector as

$$
\theta=\left[\begin{array}{llllll}
G\left(e^{j 0}\right) & G\left(e^{j \Omega}\right) & G\left(e^{-j \Omega}\right) & \ldots & G\left(e^{j \frac{n-1}{2} \Omega}\right) & G\left(e^{-j \frac{n-1}{2} \Omega}\right)
\end{array}\right]^{*}
$$

and the regressor vector as

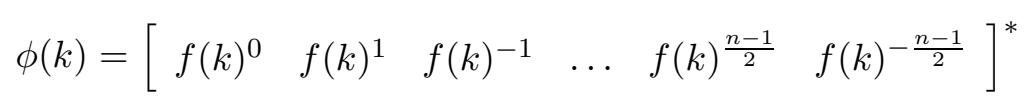

where

$$
f(k)^{r}=\frac{1}{N} \frac{1-z^{-N}}{1-e^{j r \Omega} z^{-1}}
$$

for $r=0, \pm 1, \pm 2, \ldots, \pm \frac{n-1}{2}$, and $A^{*}$ is defined as complex conjugate transpose of $A$. This allows us to rewrite equation (7) as

$$
y(k)=\phi(k)^{*} \theta+v(k)
$$

Given a set of sampled finite amount of data $\{y(1), y(2), y(3), \ldots, y(M)\}$ and $\{u(1), u(2), u(3), \ldots, u(M)\}$, we can obtain an estimate of the frequency sampling filter model using Least Squares. More specifically, we can choose $\theta$ to minimise a performance index of the form

$$
J=\sum_{k=1}^{M}\left|y(k)-\phi(k)^{*} \theta\right|^{2}
$$


If we define

$$
\Phi_{M}=\left[\begin{array}{c}
\phi(1)^{*} \\
\phi(2)^{*} \\
\phi(3)^{*} \\
\phi(4)^{*} \\
\vdots
\end{array}\right] ; Y_{M}=\left[\begin{array}{c}
y(1) \\
y(2) \\
y(3) \\
y(4) \\
\vdots
\end{array}\right]
$$

then the Least Squares estimate is

$$
\hat{\theta}=\left(\Phi_{M}^{*} \Phi_{M}\right)^{-1} \Phi_{M}^{*} Y_{M}
$$

Upon obtaining the estimate of the process frequency response parameters, the estimate of the step response at sampling instant $m$ can be expressed by

$$
\hat{g}_{m}=\sum_{i=0}^{m} \hat{h}_{i}
$$

where the estimated impulse response coefficients $\hat{h}_{0}, \hat{h}_{1}, \hat{h}_{2}, \ldots, \hat{h}_{m-1}$ are related to the estimated frequency response via

$$
\hat{h}_{i}=\frac{1}{N} \sum_{l=-\frac{n-1}{2}}^{\frac{n-1}{2}} \hat{G}\left(e^{j l \Omega}\right) e^{j l i \Omega}
$$

Substituting (12) into (11), we obtain the estimated step response coefficient at sampling instant $m$ as

$$
\hat{g}_{m}=\sum_{l=-\frac{n-1}{2}}^{\frac{n-1}{2}} \hat{G}\left(e^{j l \Omega}\right) \frac{1}{N} \frac{1-e^{j l \Omega(m+1)}}{1-e^{j l \Omega}}
$$

Note that both impulse response coefficients $\hat{h}_{i}$ and $\hat{g}_{m}$ are real because the complex frequency sampling filters are selected in complex conjugate pairs ( $n$ is an odd number). Defining

$$
Q(m)=\left[\begin{array}{llllll}
S(0, m) & S(1, m) & S(-1, m) & \ldots & S\left(\frac{n-1}{2}, m\right) & S\left(-\frac{n-1}{2}, m\right)
\end{array}\right]
$$

the estimated step response of the process at the sample $m$ is represented in a linear relation to the estimated frequency parameter vector $\theta$

$$
\hat{g}_{m}=Q(m) \hat{\theta}
$$

where $S(l, m)=\frac{1}{N} \frac{1-e^{j l \Omega(m+1)}}{1-e^{j l \Omega}}$. Also

$$
S(l, m)=\frac{1}{N}\left(1+e^{j l \Omega}+e^{j 2 l \Omega}+\ldots+e^{j m l \Omega}\right)
$$

Thus, at $l=0, S(l, m)=\frac{m+1}{N}$.

If $v($.$) is white noise with zero mean and variance \sigma^{2}$, it has been shown in Wang and Cluett (2000) that when a sufficiently large number of frequency parameter $n \leq N$ is used in the parameterisation

$$
\begin{gathered}
E\left[\hat{g}_{m}-g_{m}\right] \approx 0 \\
E\left[\left(\hat{g}_{m}-g_{m}\right)^{2}\right] \approx Q(m)\left(\Phi^{*} \Phi\right)^{-1} Q(m)^{*} \sigma^{2}=\delta(m)^{2}
\end{gathered}
$$


However, in the situation of $v($.$) being coloured noise, in order to obtain the estimation of the$ variance for the step response model, we assume that

$$
v(k)=\frac{1}{D(z)} \epsilon(k)
$$

where $D(z)=1+d_{1} z^{-1}+d_{2} z^{-2}+\ldots+d_{q} z^{-q}$, and $\epsilon(k)$ is white noise with zero mean. An iterative algorithm in the spirit of relaxed Maximum Likelihood approach (Goodwin and Payne, 1977) can be used for simultaneous estimation of the frequency sampling filter model and the autoregressive noise model. Upon convergence of the algorithm, the variance of the estimated step response at the sample instant $m$ is

$$
E\left[\left(\hat{g}_{m}-g_{m}\right)^{2}\right]=Q(m)\left(\Phi_{f}^{*} \Phi_{f}\right)^{-1} Q(m)^{*} \sigma_{f}^{2}=\delta_{f}(m)^{2}
$$

where $\Phi_{f}$ is the prefiltered data matrix, and $\sigma_{f}^{2}$ is the variance of the residuals. The estimation algorithm presented here is not only effective and well understood, but also yields unbiased step response estimate with a stochastic description of the error. With the estimated variance for the step response, statistical confidence bounds can be derived (Wang and Cluett, 2000).

\section{Continuous-time Transfer-function Identification}

As discussed in Section 3 stage two of the three-stage approach yields the system step-response in non-parametric form. As discussed in Section 3, and illustrated in Section 5, the estimated step response is relatively noise-free compared to an actual step response test and thus can be intuitively judged by the process engineer. Whilst early versions of model-based predictive control were based on step-response models, modern model-based predictive control requires a parametric model. In particular, the model-based predictive control methods being researched by the authors are set in continuous-time (Wang, 2001, Gawthrop et.al. 1998). Thus, the purpose of the third stage, discussed in this section, is to fit a continuous time parametric transfer function model to this identified step response. This transfer function model can be converted into state-space form using standard algorithms.

Consider a continuous time model described by Laplace transfer function of the form

$$
G(s)=\frac{B(s)}{A(s)}=\frac{b_{\beta} s^{\beta}+b_{\beta-1} s^{\beta-1}+\ldots+b_{0}}{s^{\alpha}+a_{\alpha-1} s^{\alpha-1}+\ldots+a_{0}}
$$

where $a_{i}, i=0, \ldots, \alpha-1$ and $b_{i}, i=0, \ldots, \beta$ are the coefficients, and $\alpha$ and $\beta$ are the orders of the numerator and denominator $(\alpha \geq \beta)$. For a given plant input signal $u(t)$, the plant output response is described by

$$
y(t)=G(p) u(t)+\eta(t)
$$

where $G(p)$ is an operator corresponding to the transfer function $G(s)$ and $\eta(t)$ is the additive continuous time disturbance. Specifically, $\eta(t)=H(p) \psi(t)$ where $H(p)$ is an operator corresponding to a transfer function $H(s)$ and $\psi(t)$ is white noise with covariance function $E[\psi(t) \psi(\tau)]=$ $\sigma_{c}^{2} \delta(t-\tau)$. By $H(p) \psi(t)$, we mean a stochastic process with power spectral density $\frac{\sigma_{c}^{2}}{2 \pi}|H(j w)|^{2}$.

In this indirect approach, the input signal to the plant is a unit step. Because the step responses are equivalent in both continuous time and discrete time cases at the sampling instant $t_{i}$, the output is the step-response resulting from stage 2 (Section 3) of our approach:

$$
y\left(t_{i}\right)=\hat{g}\left(t_{i}\right)=g\left(t_{i}\right)+\eta\left(t_{i}\right)
$$


where the disturbance $\eta\left(t_{i}\right)$ is the error contained in the estimated step response. The disturbance is a discrete sequence with known statistical properties that

$$
E\left[\eta\left(t_{i}\right)\right]=0 ; \quad E\left[\eta\left(t_{i}\right)^{2}\right]=\delta(i)^{2}
$$

If $\delta(i)$ is approximately constant for all $i$, the discrete disturbance sequence is a near white noise in discrete time. In general, $\eta($.$) has a "flat" spectrum in the low and medium frequency region and its$ amplitude is relatively small.

Suppose that an all-pole filter having denominator $C(s)=s^{\alpha+1}+c_{\alpha} s^{\alpha}+c_{\alpha-1} s^{\alpha-1}+\ldots+c_{0}$ is selected for the identification procedure. By passing both input and output measurements $u(t)$ and $y(t)$ through this filter, we obtain filtered input and output signals. This operation when applied to model (20) yields

$$
\frac{A(p)}{C(p)} y(t)=\frac{B(p)}{C(p)} u(t)+\frac{A(p)}{C(p)} \eta(t)
$$

where $A(p), B(p)$ and $C(p)$ are operators corresponding to $A(s), B(s)$ and $C(s)$ respectively. Here the filter operation is equivalent to the prefiltering operation in discrete time system save that in the continuous time case, the filter structure is restricted to all pole form.

To formulate a Least Squares problem for parameter estimation, the next step is to generate the derivatives of the filtered input and output responses. This step is simplified when a state variable filter implementation procedure is used (Gawthrop, 1984). Let $\bar{y}_{\alpha}(t), \bar{y}_{\alpha-1}(t), \ldots, \bar{y}_{0}(t)$ denote, respectively, $\frac{p^{\alpha}}{C(p)} y(t), \frac{p^{\alpha-1}}{C(p)} y(t), \ldots, \frac{1}{C(p)} y(t)$; let $\bar{u}_{\alpha}(t), \bar{u}_{\alpha-1}(t), \ldots, \bar{u}_{0}(t)$ denote, respectively, $\frac{p^{\alpha}}{C(p)} u(t), \frac{p^{\alpha-1}}{C(p)} u(t), \ldots, \frac{1}{C(p)} u(t)$. To obtain the derivatives of filtered output responses, we define a state variable vector

$$
X^{y}(t)=\left[\begin{array}{llll}
\bar{y}_{\alpha}(t) & \bar{y}_{\alpha-1}(t) & \ldots & \bar{y}_{0}(t)
\end{array}\right]^{T}
$$

Then, by choosing the state space model in a control canonical form, we have

$$
\begin{aligned}
{\left[\begin{array}{c}
\frac{d \bar{y}_{\alpha}(t)}{d t} \\
\frac{d \bar{y}_{\alpha-1}(t)}{d t} \\
\vdots \\
\frac{d \bar{y}_{0}(t)}{d t}
\end{array}\right] } & =\left[\begin{array}{cccc}
-c_{\alpha} & -c_{\alpha-1} & \ldots & -c_{0} \\
1 & 0 & \ldots & 0 \\
\vdots & & & \\
0 & \ldots & 1 & 0
\end{array}\right]\left[\begin{array}{c}
\bar{y}_{\alpha}(t) \\
\bar{y}_{\alpha-1}(t) \\
\vdots \\
\bar{y}_{0}(t)
\end{array}\right]+\left[\begin{array}{c}
1 \\
0 \\
\vdots \\
0
\end{array}\right] y(t) \\
& =V\left[\begin{array}{c}
\bar{y}_{\alpha}(t) \\
\bar{y}_{\alpha-1}(t) \\
\vdots \\
\bar{y}_{0}(t)
\end{array}\right]+W y(t)
\end{aligned}
$$

where $V$ and $W$ are the corresponding system matrices. The solution of the state space equation (23), assuming zero initial conditions, gives the derivatives of the filtered output responses. Similarly, define

$$
X^{u}(t)=\left[\begin{array}{ll}
\bar{u}_{\alpha}(t) & \bar{u}_{\alpha-1}(t) \ldots \bar{u}_{0}(t)
\end{array}\right]^{T}
$$

Then

$$
\begin{aligned}
& \dot{X}^{u}(t)=V X^{u}(t)+W u(t) \\
& X^{u}(0)=0_{\alpha}
\end{aligned}
$$

where $0_{n}$ is a zero column vector of length $n$. 
At this stage, although the state variables are expressed in the continuous time form, the inputs to the state variable filters are in the form of discrete measurement. The state equations are required to be solved numerically to give the derivatives of the filtered input and output signals. One of the simple approaches to obtain the filtered responses is given as follows. The response of the state variable $X(t)$ for $t_{i} \leq t \leq t_{i+1}$ is

$$
\int_{t_{i}}^{t_{i+1}} d X(t)=\int_{t_{i}}^{t_{i+1}}(V X(t)+W u(t)) d t
$$

which is

$$
X\left(t_{i+1}\right) \approx X\left(t_{i}\right)+\frac{V X\left(t_{i}\right)+W u\left(t_{i}\right)+V X\left(t_{i+1}\right)+W u\left(t_{i+1}\right)}{2} \Delta t
$$

Thus, the solution of the state variable vector is, by given initial condition $X\left(t_{0}\right), i=1,2,3, \ldots$,

$$
X\left(t_{i+1}\right)=\left(I-\frac{\Delta t}{2} V\right)^{-1}\left(I+\frac{\Delta t}{2} V\right) X\left(t_{i}\right)+\left(I-\frac{\Delta t}{2} V\right)^{-1} \frac{\Delta t}{2} W\left(u\left(t_{i}\right)+u\left(t_{i+1}\right)\right)
$$

Because Trapezoidal rule is used in the approximation of the integral equation (25), the approximation error between two sample points is, $t_{i} \leq t \leq t_{i+1}$

$$
E_{c}=-\frac{1}{12} \Delta t^{3}\left(V \frac{d^{2} X(t)}{d t^{2}}\right)+W \frac{d^{2} u(t)}{d t^{2}}
$$

The approximation scheme is stable if

$$
\lambda_{\max }\left[\left(I-\frac{\Delta t}{2} V\right)^{-1}\left(I+\frac{\Delta t}{2} V\right)\right]<1
$$

where $\lambda_{\max }(A)$ is the maximum eigenvalue of $A$. With the discretised state variables defined by (23)-(24), equation (22) can be written as

$$
\bar{y}_{\alpha}\left(t_{i}\right)+a_{\alpha-1} \bar{y}_{\alpha-1}\left(t_{i}\right)+\ldots+a_{0} \bar{y}_{0}\left(t_{i}\right)=b_{\beta} \bar{u}_{\beta}\left(t_{i}\right)+b_{\beta-1} \bar{u}_{\beta-1}\left(t_{i}\right)+\ldots+b_{0} \bar{u}_{0}\left(t_{i}\right)+\eta_{f}\left(t_{i}\right)
$$

where $\eta_{f}\left(t_{i}\right)$ denotes the filtered disturbance variable. To estimate the parameters $a_{i}, i=0,1,2$ $\ldots \alpha-1, b_{i}, i=0,1, \ldots, \beta$, equation (30) is reformulated into a standard regression form as

$$
\bar{y}_{\alpha}\left(t_{i}\right)=\phi\left(t_{i}\right)_{c}^{T} \theta_{c}+\eta_{f}\left(t_{i}\right)
$$

where

$$
\phi\left(t_{i}\right)_{c}^{T}=\left[\begin{array}{lll}
-\bar{y}_{\alpha-1}\left(t_{i}\right) & \ldots-\bar{y}_{0}\left(t_{i}\right) \bar{u}_{\alpha}\left(t_{i}\right) \ldots \bar{u}_{0}\left(t_{i}\right)
\end{array}\right]
$$

and

$$
\theta_{c}^{T}=\left[\begin{array}{lllll}
a_{\alpha-1} & \ldots & a_{0} & b_{\beta} & \ldots \\
b_{0}
\end{array}\right]
$$

The Least Squares solution for the set of parameters in the continuous time model is given by

$$
\theta_{c}=\left(\sum_{i=0}^{N-1} \phi\left(t_{i}\right) \phi\left(t_{i}\right)^{T}\right)^{-1} \sum_{i=0}^{N-1} \phi\left(t_{i}\right) \bar{y}_{\alpha}\left(t_{i}\right)
$$

The continuous time parameter vector $\theta_{c}$ can also be estimated using the multiple model estimation algorithm (Wang and Gawthrop, 2001). 


\section{Experimental Evaluation}

Extrusion is a continuous process in which a rotating screw is used to force the food material through the barrel of the machine and out through a narrow die opening. In this process the material is simultaneously transported, mixed, shaped, stretched and sheared under elevated temperature and pressure. Figure 4 shows the block diagram of a twin screw food extruder. The extruder in the study is an APV-MPF40 co-rotating twin-screw extruder. The extruder has the following specifications:

- Throughput: $20-75 \mathrm{~kg} / \mathrm{hr}$

- Screw Diameter: $40 \mathrm{~mm}$

- Length to Diameter Ratio: 25:1

- Feeding System: Gravimetric

- Process Monitoring: via Siemens 95U PLC

The extruder was run with the following setup for all experiments:

- Screw Profile No: 12 (High Shear Configuration)

- Die: $3 \mathrm{~mm}$ diameter, $2 \mathrm{~mm}$ land length

The factors which can be adjusted on-line for control purpose include the screw speed, the rate at which the raw material enters the extruder (termed feed rate) and the liquid injection rate (measured via liquid pump speed). The process output variables which are important for product quality control (Wang et al. 2001) are the Specific Mechanical Energy, Die Pressure, screw motor torque, and Product Temperature at the location which is close to the die. All these variables can be measured on-line by using sensors.

This experiment apparatus has been implemented on an extrusion plant through the existing Supervisory Control and Data Acquisition system on the extruder, and has been successfully used in collecting experimental data for identification purposes for all input and output variables under investigation. Figure 5 shows the user-interface of relay feedback control system for the identification experiment.

\subsection{Data Acquisition using Relay Feedback}

The basic procedure in the identification experiment is to perturb the input signals one at a time and measure the responses in all output signals. The perturbation of the input signal is generated automatically by a nonlinear relay feedback mechanism. In other words, when one relay feedback control system is in operation, the rest of the input signals remains constant. Since the extruder is not a single input and single output system, the first issue in the design of the experiment is pairing of input and output variables for the relay feedback control systems. This issue is addressed by paring the input variable with the output variable that has the largest time constant, also by taking consideration of the extruder physics. This approach ensures that sufficient low frequency components exist in the excitation signals for all output variables. More specifically, screw speed is used to manipulate specific mechanical energy, liquid pump speed is used to manipulate motor torque, feed rate is used to manipulate die pressure, zone 9 temperature setpoint is used to manipulate the temperature at the die.

The relay feedback control apparatus has been used in collecting experimental data for identification. This particular set of experimental data analysed here was generated by controlling die pressure with material feedrate. Figure 6 shows the input signal generated by the relay apparatus and the responses of SME, motor torque and die pressure. 


\subsection{Step Response using Frequency-sampling Filters}

Three step response models with confidence bounds were estimated where the relaxed maximum likelihood method (Goodwin and Payne, 1977) was used to iteratively identify three noise models respectively. The model orders and the converged noise models are given below, respectively, for SME, Motor Torque and Die Pressure. A sufficiently large $n$ was chosen for the three cases to ensure the unmodelled dynamics were negligible.

- SME: $N=180, n=119$ and $D(z)=1-0.4757 z^{-1}-0.3543 z^{-2}$

- Motor Torque: $N=180, n=119$ and $D(z)=1-0.7942 z^{-1}-0.0843 z^{-2}$

- Die Pressure: $N=280, n=119$ and $D(z)=1-1.5477 z^{-1}+0.1617 z^{-2}+0.6247 z^{-3}-$ $0.1083 z^{-4}-0.095 z^{-5}$

where the noise model orders were selected using the PRESS criterion given in Wang and Cluett (1996). The step response models with confidence bounds are shown in Figures 7-9. The raw experimental data shown in Figure 6 are compressed into three individual step response models. In this application, the raw data set contains 1800 samples for each pair of physical variables whilst the maximum number of samples in the step response models is 280 (Die Pressure has a long settling time). It is seen from these Figures that the confidence bounds are relatively large, particularly in the steady state parts of the responses, which indicates the plant disturbances have frequency contents that are concentrated in the low frequency regions. Essentially, the confidence bounds for steady state correspond to the variance of the estimate $G\left(e^{j 0}\right)$ in the frequency sampling filter model (Wang and Cluett, 2000).

\subsection{Continuous-time Transfer-function Identification}

Upon obtaining the continuous time step response data, the identification algorithm with model structure selection given in Wang and Gawthrop (2001) was used in the estimation of a continuous time transfer function model. Both sum of squares of step response errors $E_{s}=\sum_{k=1}^{N-1}\left[\hat{g}\left(t_{k}\right)-\right.$ $\left.g\left(t_{k}\right)\right]^{2}$ and the sum of squares of prediction errors $E_{p}=\sum_{k=1}^{M}\left|y\left(t_{k}\right)-\frac{B(p)}{A(p)} u\left(t_{k}\right)\right|^{2}$ were used for model structure determination. The relative model order is selected to be one for the three cases; and the denominators of the state variable filters are selected to be in the form of $C(s)=(s+\gamma)^{5}$, where $\gamma=0.12,0.12$ and 0.012 respectively for SME, Motor Torque and Die Pressure. The model errors are summarized in Tables (5.3)-(5.3). Based on these Tables, the model orders are selected and the estimated continuous time transfer functions are given below:

SME

$$
G(s)=\frac{10 \times 10^{-3}\left(1.68 s^{3}-0.0396 s^{2}-0.0312 s-0.001\right)}{s^{4}+0.1119 s^{3}+0.0195 s^{2}+5.78 \times 10^{-4} s+2.4 \times 10^{-5}}
$$

Motor Torque

$$
G(s)=\frac{0.168 \times 10^{-3} s^{2}-0.345 \times 10^{-4} s-2.34 \times 10^{-7}}{s^{3}+0.1402 s^{2}+0.7733 \times 10^{-2} s+0.1305 \times 10^{-3}}
$$

Die Pressure

$$
G(s)=\frac{-1.332 \times 10^{-3} s^{3}+3.144 \times 10^{-4} s^{2}-2.2011 \times 10^{-5} s+1.134 \times 10^{-7}}{s^{4}+0.108 s^{3}+9.59 \times 10^{-3} s^{2}+3 \times 10^{-4} s+5.13 \times 10^{-6}}
$$

The step responses of the continuous time models are compared to their respective responses in Figures 7- 9. In addition, the predicted output responses using the continuous time models are compared with experimental data shown in Figures 10-12. 


\begin{tabular}{|c|c|c|c|}
\hline Model Order & SME & Motor Torque & Die Pressure \\
\hline$\alpha=1$ & 0.0562 & $3.11 \times 10^{-4}$ & 0.3793 \\
\hline$\alpha=2$ & 0.007 & $2.52 \times 10^{-5}$ & \\
\hline$\alpha=3$ & 0.0017 & $7.17 \times 10^{-6}$ & 0.0152 \\
\hline$\alpha=4$ & 0.0015 & $6.41 \times 10^{-6}$ & 0.0045 \\
\hline$\alpha=5$ & 0.0014 & $6.8 \times 10^{-6}$ & 0.0043 \\
\hline
\end{tabular}

Table 1: Sum of Squares of Step Response Errors

\begin{tabular}{|c|c|c|c|}
\hline Model Order & SME & Motor Torque & Die Pressure \\
\hline$\alpha=1$ & $2.09 \times 10^{6}$ & $8.9 \times 10^{3}$ & $5.25 \times 10^{6}$ \\
\hline$\alpha=2$ & $1.09 \times 10^{6}$ & $4.01 \times 10^{3}$ & \\
\hline$\alpha=3$ & $6.64 \times 10^{5}$ & $2.69 \times 10^{3}$ & $2.39 \times 10^{6}$ \\
\hline$\alpha=4$ & $6.38 \times 10^{5}$ & $2.65 \times 10^{3}$ & $1.77 \times 10^{6}$ \\
\hline$\alpha=5$ & $6.73 \times 10^{5}$ & $2.81 \times 10^{3}$ & $1.75 \times 10^{6}$ \\
\hline
\end{tabular}

Table 2: Sum of Squares of Prediction Errors

\section{Conclusions}

This paper has presented a three-stage approach to system identification in the continuous-time which is appropriate for day-to-day application by plant engineers in the process industry. The three stages are: data acquisition using relay feedback; non-parametric identification of the system step response; and parametric model fitting of the identified step-response. One of the contributions of the paper is to combine them in a novel way to provide an indirect continuous time identification method. Within this framework, discrete time noise model is naturally adopted into continuous time system identification.

The method has been evaluated on a pilot-scale food-cooking extruder. As discussed in Section 5 , each stage has a clear intuitive output which enhances the confidence of the process engineer in the final result.

Comparison results using different continuous time identification methods for this set of food extruder data are currently under investigation. The results will be reported in a future work (Wang, Young and Gawthrop, 2003).

\section{References}


see enclosed document drawing figure1.doc

Figure 1: Data Acquisition: Relay Feedback 


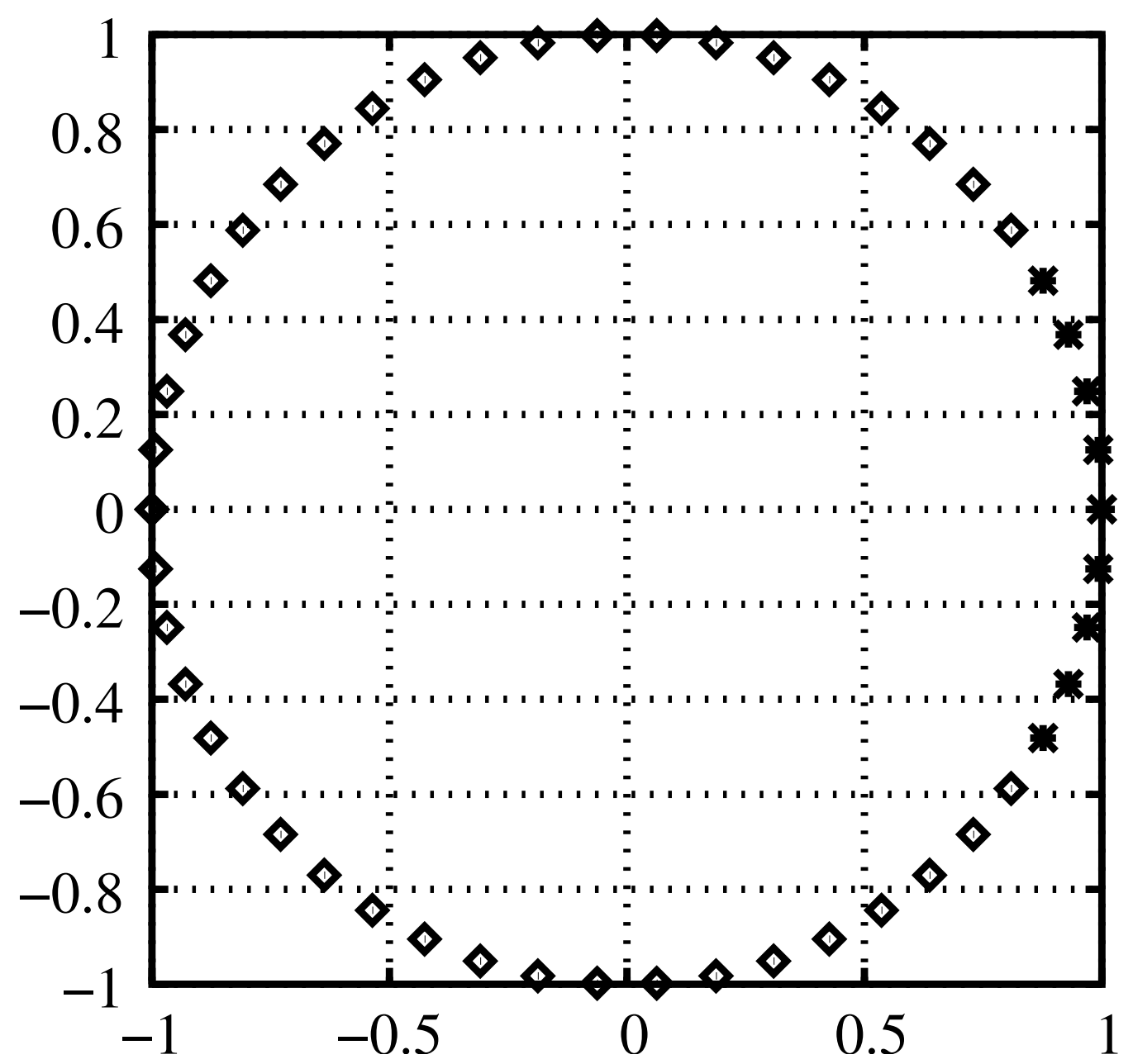

Figure 2: Frequency-sampling filter $n=9, N=50: \star$ : FSF poles; $\diamond:$ unit circle 


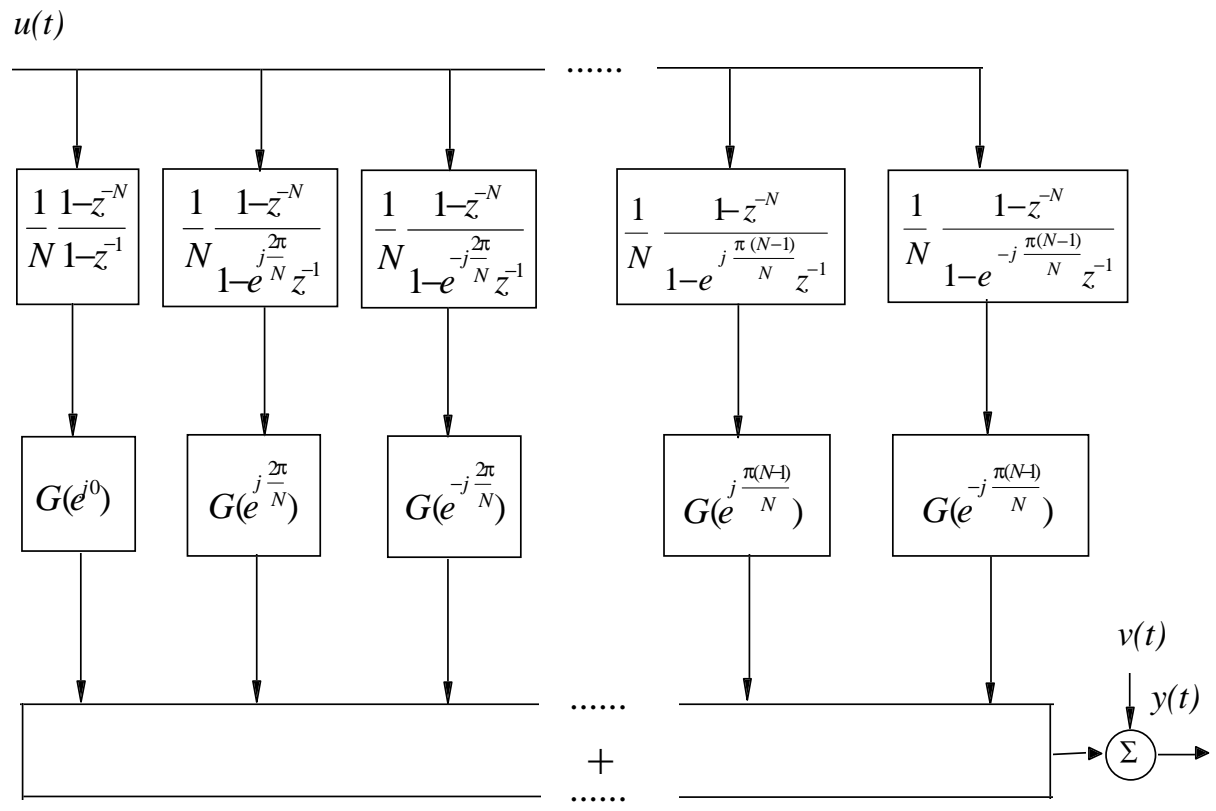

Figure 3: Block Diagram of Frequency-sampling filter structure 
see enclosed word document drawing figure4.doc

Figure 4: Schematic of the Food Extruder 
see enclosed word document drawing

Figure 5: Data Acquisition: User Interface 

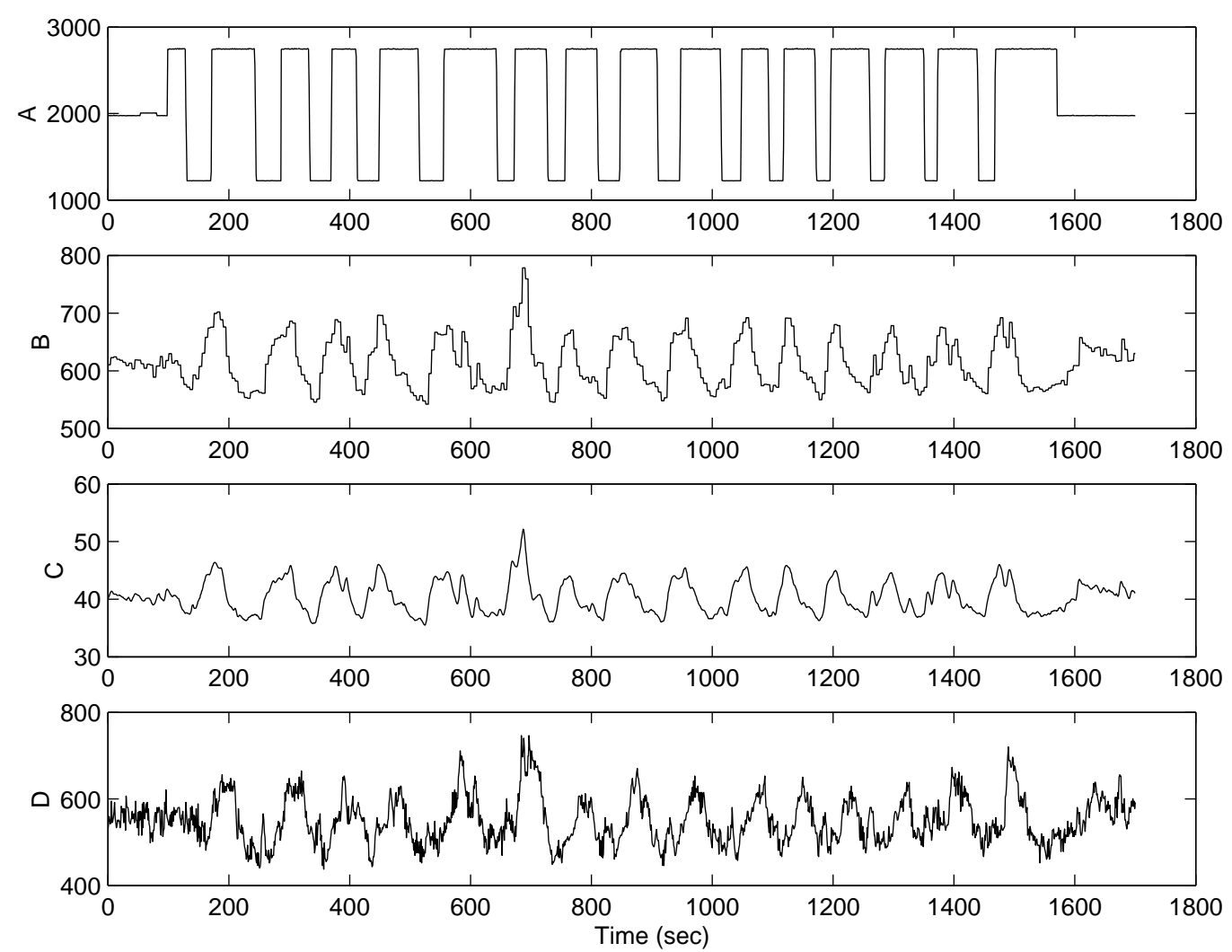

Figure 6: Experimental data. From top to bottom: A. feedrate; B. SME; C. Motor torque; D. Die Pressure 


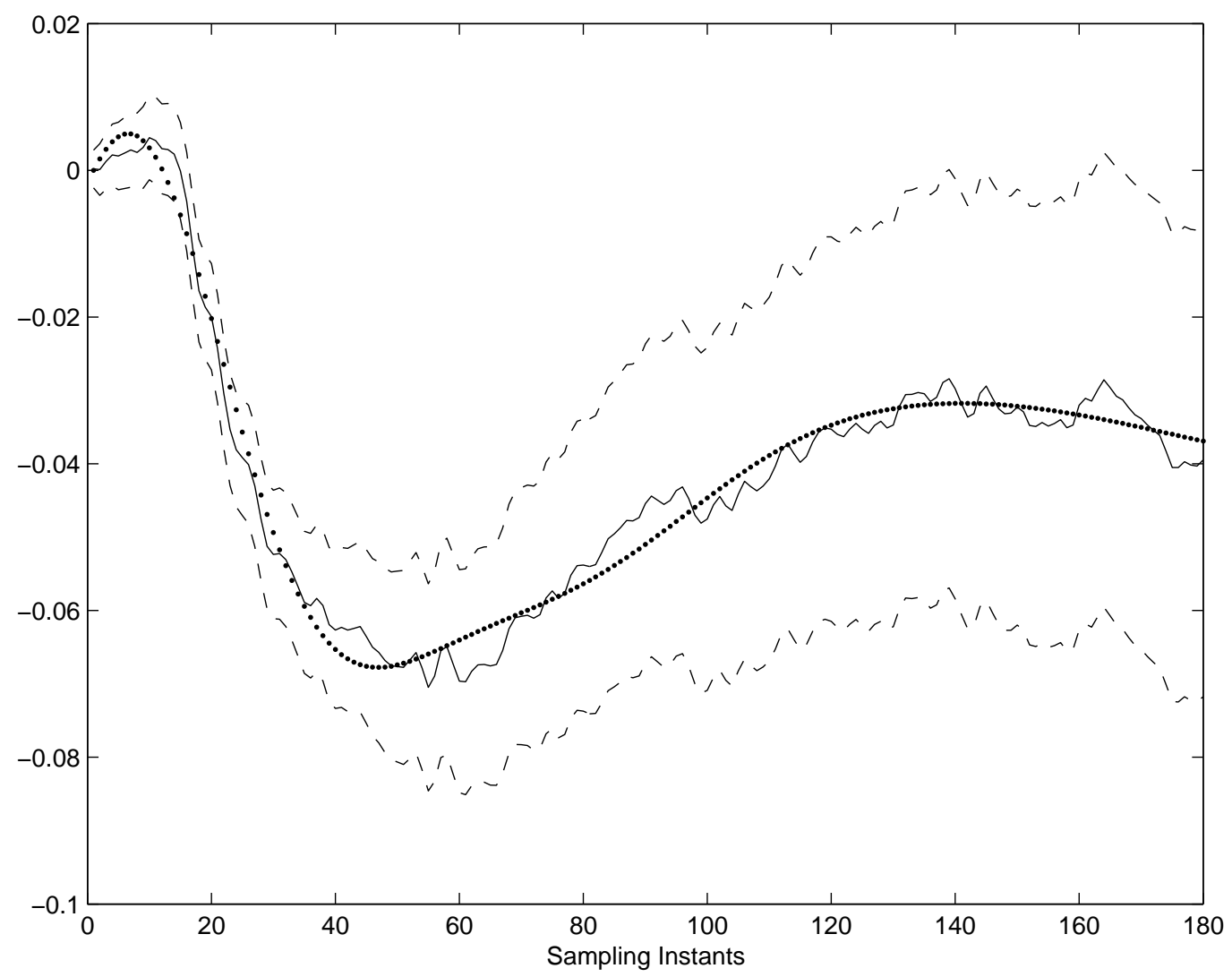

Figure 7: Step response of SME. Solid line-estimated step response with $2 \sigma$ confidence bounds; Dark dotted line-step response from the continuous time model. 


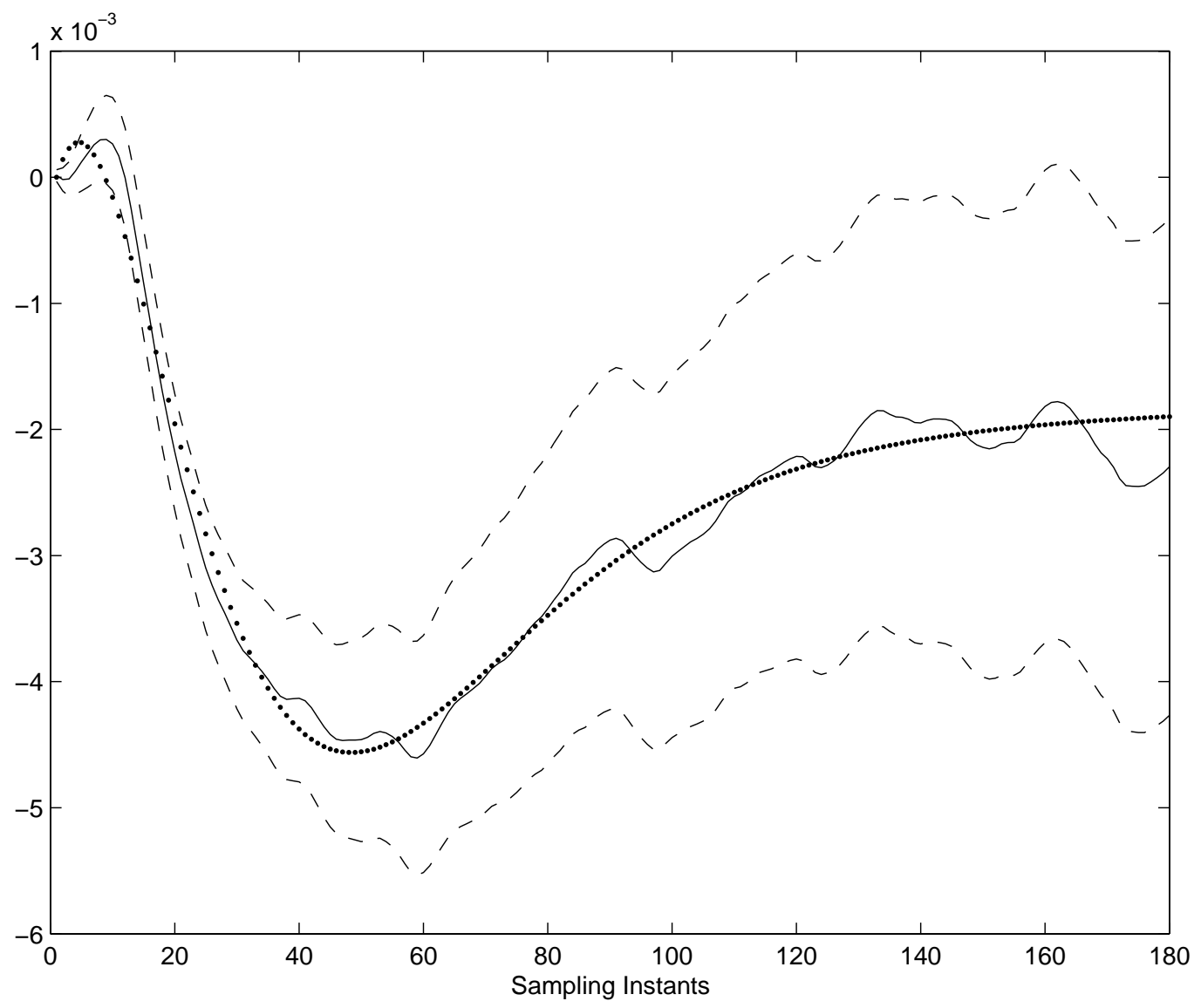

Figure 8: Step response of Motor Torque. Solid line-estimated step response with $2 \sigma$ confidence bounds; Dark dotted line-step response from the continuous time model. 


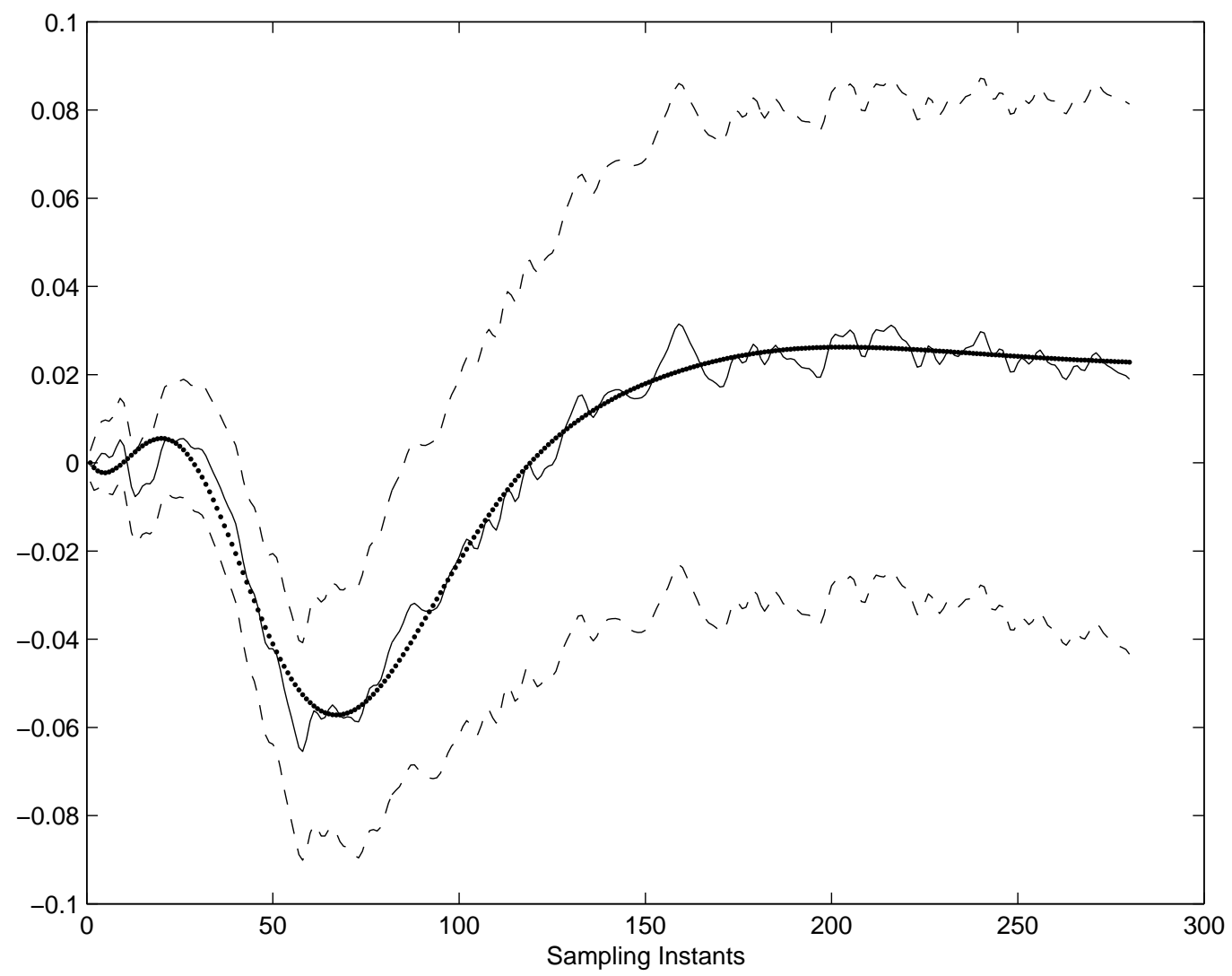

Figure 9: Step response of Die Pressure. Solid line-estimated step response with $2 \sigma$ confidence bounds; Dark dotted line-step response from the continuous time model. 


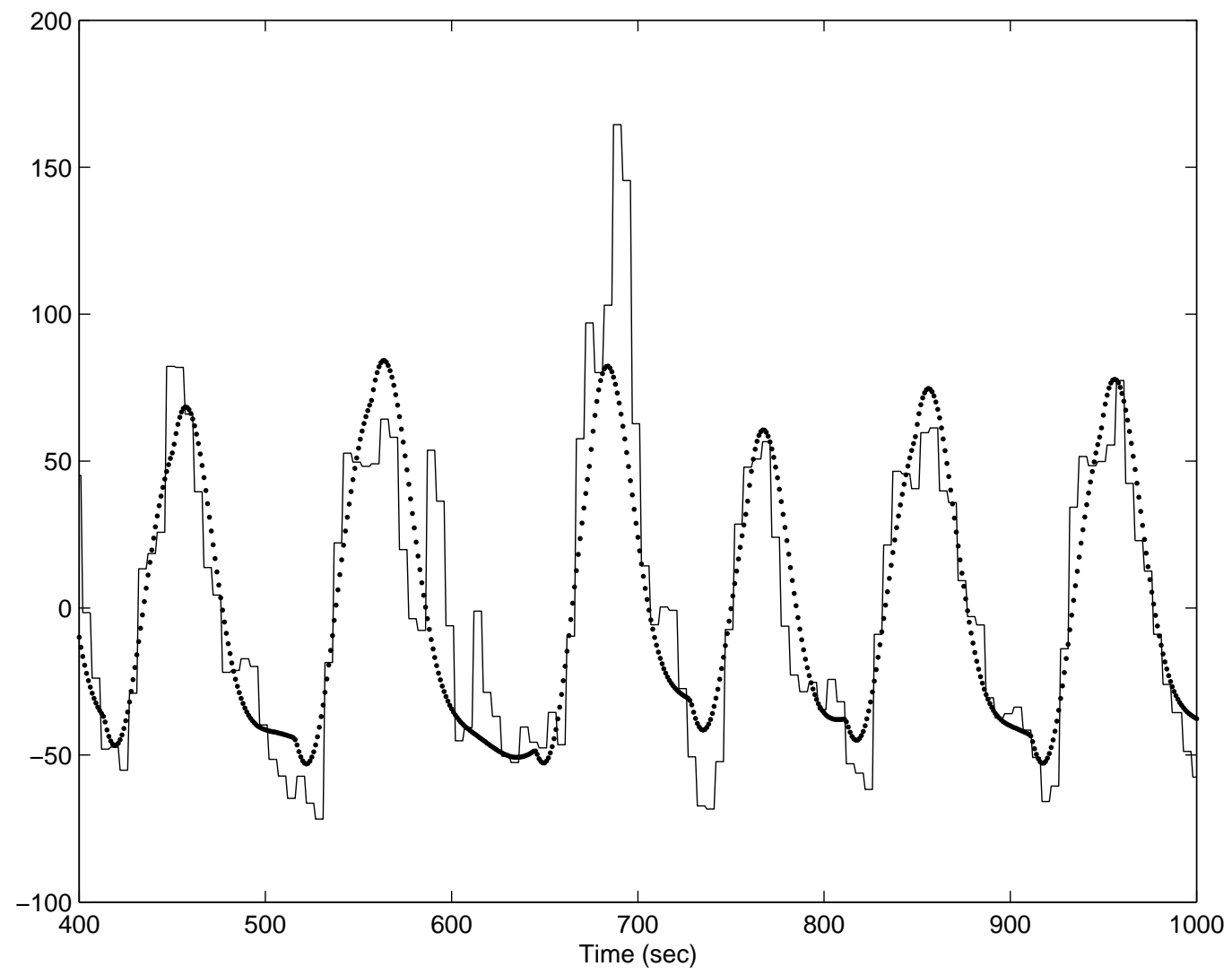

Figure 10: Predicted and actual SME. Solid line-actual data; Dark dotted line-predicted response from the continuous time model. 


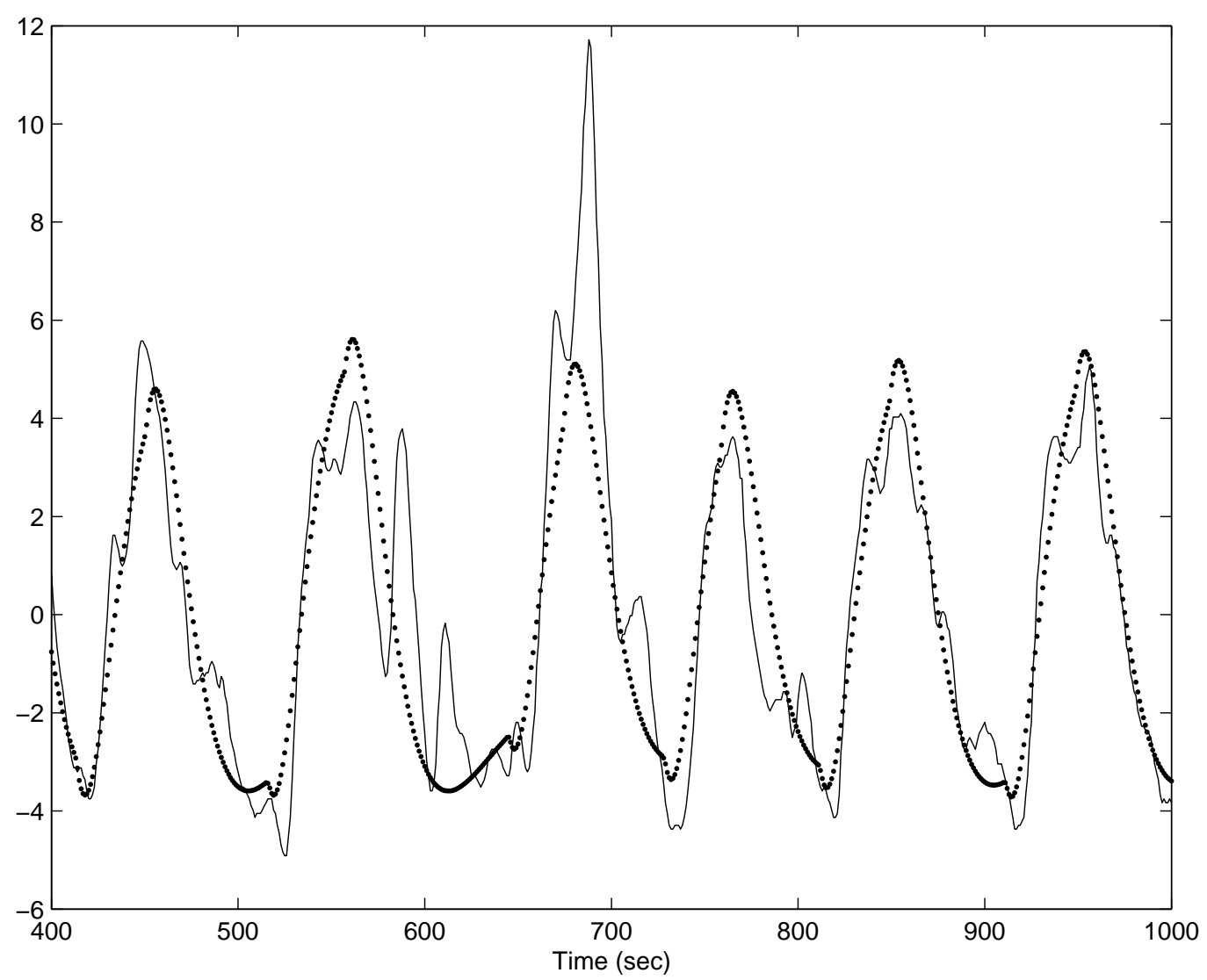

Figure 11: Predicted and actual Motor Torque. Solid line-actual data; Dark dotted line-predicted response from the continuous time model. 


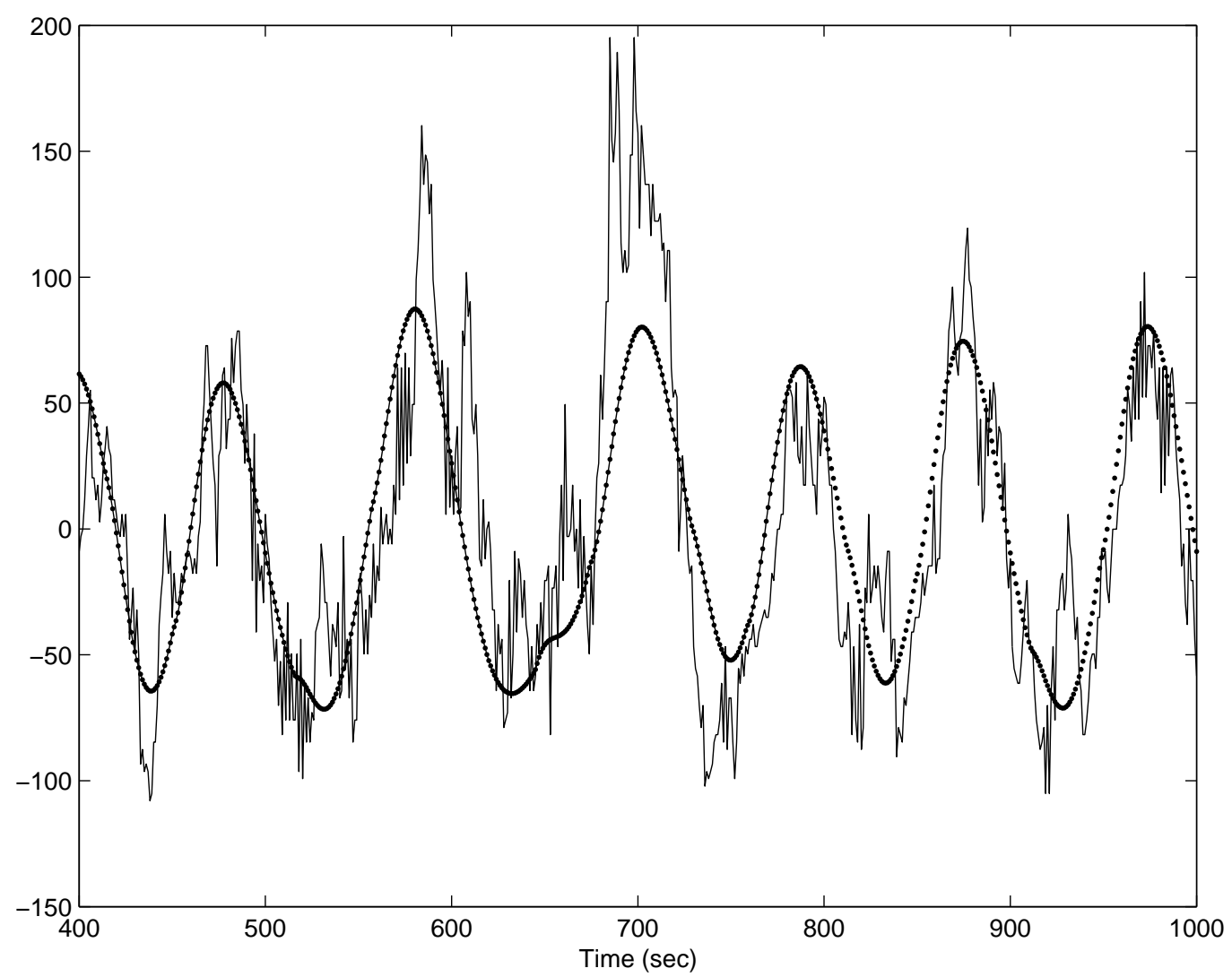

Figure 12: Predicted and actual Die Pressure. Solid line-actual data; Dark dotted line-predicted response from the continuous time model. 